\title{
Temporal Characterization of Front-End for Yb-Based High-Energy Optical Waveform Synthesizers
}

\author{
Hüseyin Çankaya ${ }^{1,2}$, Anne-Laure Calendron ${ }^{1,2,3}$, Giovanni Cirmi ${ }^{1,2}$, Chun Zhou ${ }^{1,3}$, Oliver D. Mücke ${ }^{1,2}$, \\ and Franz X. Kärtner ${ }^{1,2,3,4}$ \\ ${ }^{1}$ Center for Free-Electron Laser Science, Deutsches Elektronen Synchrotron (DESY) Notkestrasse 85, 22607 Hamburg, Germany \\ ${ }^{2}$ The Hamburg Centre for Ultrafast Imaging, University of Hamburg, Luruper Chaussee 149, 22761 Hamburg, Germany \\ ${ }^{3}$ Department of Physics, University of Hamburg, Luruper Chaussee 149, 22761 Hamburg, Germany \\ ${ }^{4}$ Research Laboratory of Electronics, Massachusetts Institute of Technology, Cambridge, Massachusetts 02139, USA \\ Authore-mail address: huseyin.cankaya@cfel.de
}

\begin{abstract}
We demonstrate temporal characterization of the front-end for an Yb-based, passively CEP-stable, two-octave-wide, two-channel optical parametric synthesizer driven by slightly subpicosecond pump pulses from a multi-mJ regenerative amplifier at $1 \mathrm{kHz}$.
\end{abstract}

OCIS codes: (320.6629) Supercontinuum generation; (320.7110) Ultrafast nonlinear optics; (190.7110) Ultrafast nonlinear optics

High-harmonic generation (HHG) is an established powerful method, which allows characterization of atomic or interatomic transitions below the femtosecond time scale in the extreme ultraviolet (XUV) and soft X-ray spectral regions. However, HHG is a very inefficient process to generate isolated attosecond pulses. Coherent optical pulse synthesis of high-energy, few-cycle pulses is one of the most promising methods to efficiently generate isolated attosecond pulses because of the flexibility in spectral shaping and scalability in spectral bandwidth as well as energy [1-4]. Since the HHG process is driven by the electric field $E(t)$ of an optical pulse, control of the carrierenvelope phase (CEP) is crucial. The CEP can be controlled either actively [2], requiring several feedback loops increasing the system's complexity, or passively, using the difference-frequency process [3-5].

Previously, we demonstrated passively CEP-stabilized synthesizers pumped by Ti:Sapphire laser technology [3,4], and we have been pursuing average-power scaling with Yb-based pump technology [6,7]. In this particular study, we demonstrate the temporal characterization of a two-channel parametric synthesizer at the $\mu \mathrm{J}$ energy level, which will serve as the seed source for a mJ-level pulse synthesizer. The system comprises two-octave, passively CEP-stable, two-channel optical parametric amplifiers (OPAs) driven by sub-picosecond pulses [8] from an Ybbased multi-mJ regenerative amplifier. The OPAs are seeded by a passively CEP-stable front-end based on whitelight supercontinuum generation in bulk [9].

Fig. 1 shows the layout of the dual-channel OPA system driven by an Yb:KYW regenerative amplifier. The compressed output of the $\mathrm{Yb}: \mathrm{KYW}$ regenerative amplifier delivering 4.2-mJ, 615-fs long pulses [3] is split between a front-end generating nJ-level, CEP-stable pulses with spectrum extending from the visible up to the Mid-IR, and the pumping of two 2-stage OPA channels in the Near and Mid-IR spectral regions. The CEP-stable front-end comprises 3 steps: a first white-light stage broadens the spectrum of the regenerative amplifier; 2 consecutive OPA stages amplify a narrow-band spectral region to the $\mu \mathrm{J}$ level in the Mid-IR; the second harmonic of the resulting CEP-stable idler generates white-light in YAG, which is the seed of the following OPAs $[3,4]$.

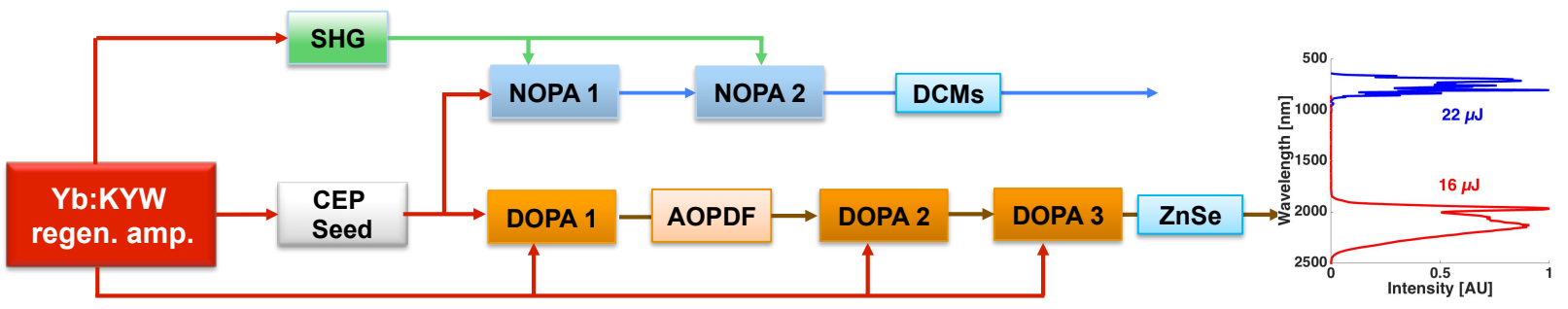

Fig. 1. Layout of the dual-channel OPA system driven by an Yb:KYW regenerative amplifier: the signal is generated in the CEP-stable white-light based front-end, and spectra after amplification and compression stages are shown.

The Near-IR non-collinear OPA (NOPA) stages are pumped by the second harmonic of the regenerative amplifier at $515 \mathrm{~nm}$ with $5.7 \mu \mathrm{J}$ (NOPA1) and $132 \mu \mathrm{J}$ (NOPA2) and resulted in an output energy of $24 \mu \mathrm{J}$ at the end of the second stage without any superfluorescence. The amplified spectrum extends 640 and $960 \mathrm{~nm}$ supporting 7.7- 
fs (FWHM) transform-limited pulses. The residual dispersion of the amplified pulses is compensated by employing double-chirped mirror (DCM) pairs designed for octave-spanning Ti:Sapphire oscillators.

The Mid-IR degenerate OPA (DOPA) stages are pumped at $1030 \mathrm{~nm}$ with $95 \mu \mathrm{J}$ (DOPA1), $35 \mu \mathrm{J}$ (DOPA2), $570 \mu \mathrm{J}$ (DOPA3) pulse energies. For precision dispersion control, an Acousto-Optic Programmable Dispersive Filter (AOPDF) is implemented between the first and second stages accompanied by a 2-mm YAG plate, which provides negative dispersion. The second DOPA stage is employed to compensate for the losses due to AOPDF. At the end of the third-stage DOPA, the pulses are amplified to $16 \mu \mathrm{J}$. The spectrum is extending the range from $1840-2460 \mathrm{~nm}$, and supports 28.4-fs pulses. Then the pulses are compressed inside two anti-reflection-coated ZnSe wedges with propagation distance of $1.5 \mathrm{~mm}$.

Fig. 2 shows the temporal characterization of the pulses in the Near-IR (top row) and Mid-IR (bottom row) channels by using the second-harmonic FROG technique. In the Near-IR, the compression is achieved down to 12.1 fs (full width at half maximum, FWHM) by using 5 DCM pairs, while the transform-limited pulse duration is $7.7 \mathrm{fs}$. The corresponding FROG retrieval error is $0.81 \%$. After compression, the pulse energy is reduced from 24 to $22 \mu \mathrm{J}$ due to losses of the imaging metallic optics. The spectral phase in the short wavelength region is not compensated because of the limited-capability of the DCMs in this region. The compression can be further improved by using either custom-designed DCMs or a pulse shaper in the Near-IR. On the other hand, the AOPDF adaptively controls the dispersion of the Mid-IR channel. As can be seen from the SHG-FROG results (Fig. 2 second row), the spectral phase is almost constant over the full spectral range, which results in a pulse duration of $31.1 \mathrm{fs}$, close to its transform-limit (28.4 fs). The corresponding FROG error is $0.16 \%$.
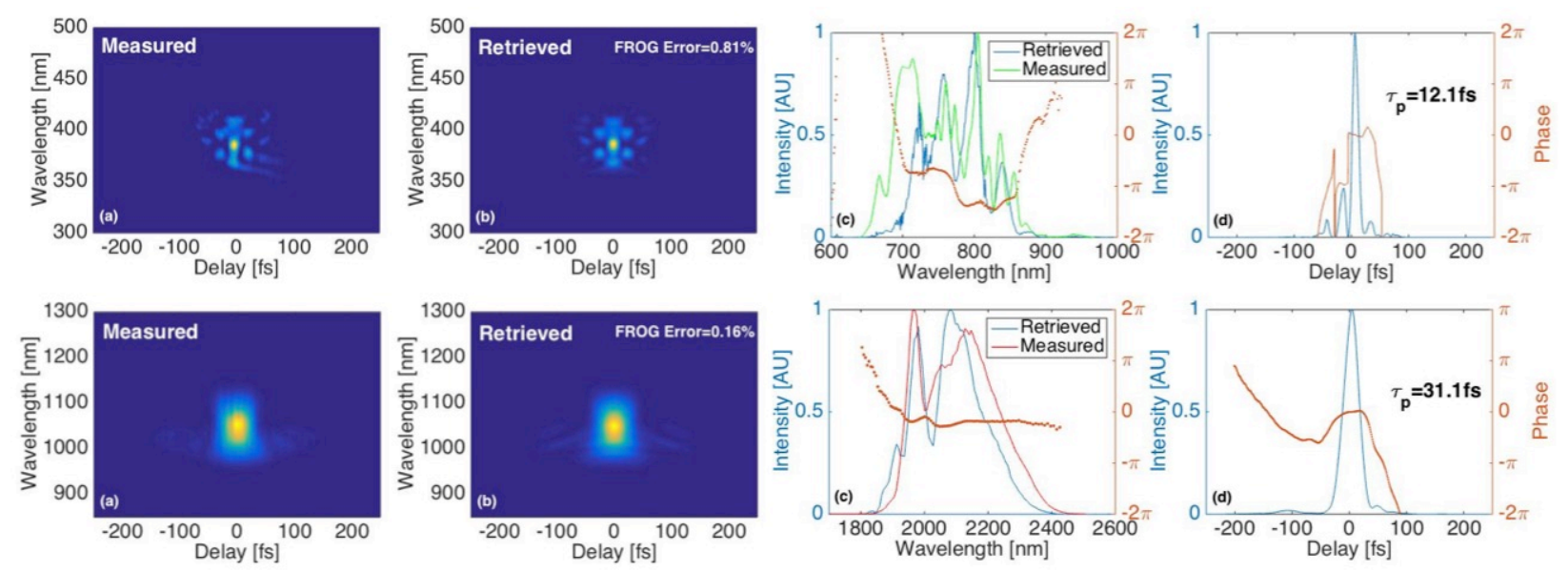

Fig. 2 . SHG-FROG characterization of the OPA outputs from the two channels. Near-IR NOPA (top row) and Mid-IR channels (bottom) are shown. (a) Measured and (b) retrieved FROG traces. (c) Measured spectrum, retrieved spectral intensity and phase. (d) Retrieved temporal intensity and phase profiles. The retrieved FWHM pulse durations are indicated.

In summary, we demonstrate the temporal characterization of a passively CEP-stable two-octave-wide pulsed light source with $\mu \mathrm{J}$ energy levels. The NIR channel is compressed down to $12.1 \mathrm{fs}$, while the IR channel is compressed to $31.2 \mathrm{fs}$. Our current aim is to scale up the energy with further OPA stages employing cryogenically cooled high-energy Yb-based pump amplifiers and combine them coherently to obtain sub-cycle pulses. This is an ideal seed source for a two-octave-wide optical waveform synthesizer with high-energy and high repetition rate.

\section{References}

[1] A. Wirth et al., "Synthesized Light Transients," Science 34, 195 (2011)

[2] S.-W. Huang et al., "High-energy pulse synthesis with sub-cycle waveform control for strong-field physics," Nature Photonics 5, 475 (2011)

[3] G. M. Rossi et al., "Spectro-Temporal Characterization of All Channels in a Sub-Optical-Cycle Parametric Waveform Synthesizer," CLEO San Diego, (2014), SF1E.3

[4] O. D. Mücke et al., "Toward Waveform Nonlinear Optics Using Multimillijoule Sub-Cycle Waveform Synthesizers," IEEE JSTQE 21, 8700712 (2015).

[5] G. Cerullo, A. Baltuška, O. D. Mücke, C. Vozzi, "Few-optical-cycle light pulses with passive carrier-envelope phase stabilization,“" Laser \& Photonics Reviews 5, 323-351 (2011).

[6] H. Cankaya et al., "Front-End of Yb-based High-Energy Optical Waveform Synthesizer," CLEO, San Jose (2015), SW4O.7.

[7] A.-L. Calendron et al., "Towards dual-channel Yb-pumped broadband OPCPA synthesizer, "CLEO Europe, Munich (2015), CF.9.1.

[8] A.-L. Calendron, H. Cankaya, F.X. Kärtner, "High-energy kHz Yb:KYW dual-crystal regenerative amplifier," Optics Express 22,24752 (2014).

[9] H. Cankaya, A.-L. Calendron, F. X. Kärtner, " Passively CEP-stable front end for frequency synthesis, “ UP, Okinawa, (2014), 07.Mon.P1.58 